\title{
Novel Triazine-Based Covalent Organic Framework as a Superadsorbent for the Removal of Mercury(II) from Aqueous Solutions
}

\author{
Mohaddeseh Afshari, Mohammad Dinari," Kiomars Zargoosh, ${ }^{*}$ Hossein Moradi
}

\section{Corresponding Authors}

Mohammad Dinari - Department of Chemistry, Isfahan

University of Technology, Isfahan 84156-83111, Islamic

Republic of Iran; orcid.org/0000-0001-5291-7142;

Phone: +98-31-3391-3270; Email: dinari@iut.ac.ir,

mdinary@gmail.com; Fax: +98-31-3391-2350

Kiomars Zargoosh - Department of Chemistry, Isfahan

University of Technology, Isfahan 84156-83111, Islamic

Republic of Iran; Phone: +98-31-3391-3287;

Email: Kiomarszargoosh@iut.ac.ir; Fax: +98-31-3391-2352

Authors

Mohaddeseh Afshari - Department of Chemistry, Isfahan

University of Technology, Isfahan 84156-83111, Islamic

Republic of Iran

Hossein Moradi - Department of Chemistry, Isfahan University

of Technology, Isfahan 84156-83111, Islamic Republic of Iran 


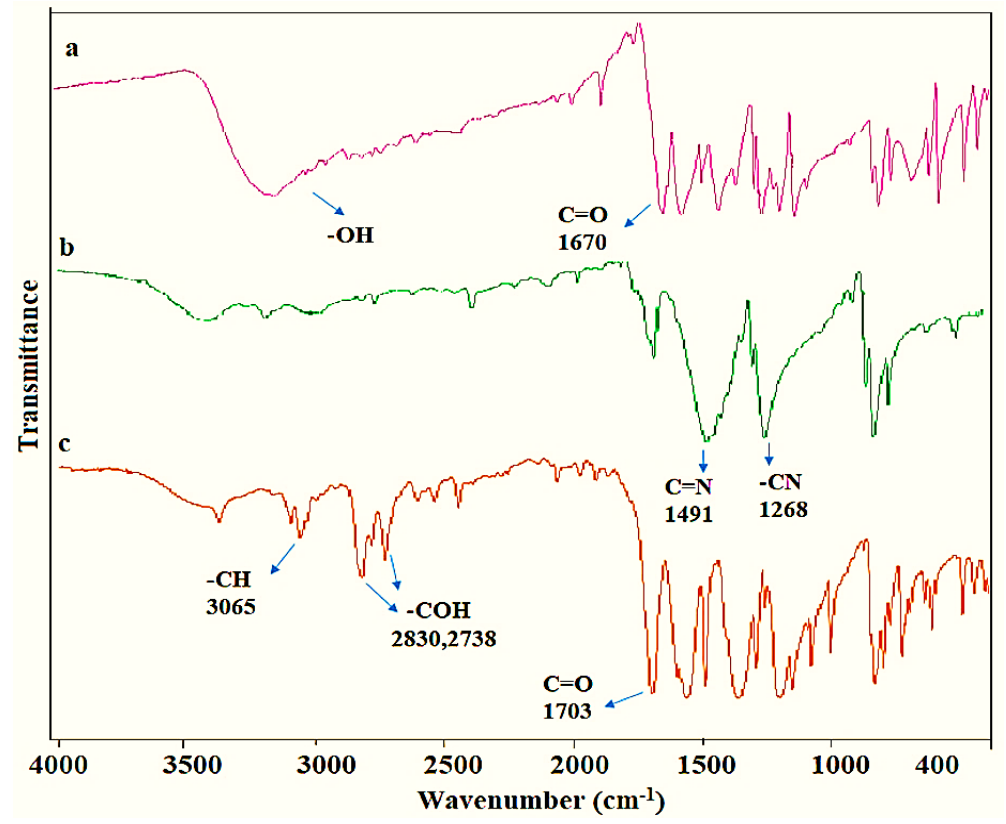

Figure S1. FT-IR spectra of (a) p-hydroxy benzaldehyde, (b) CC, and (c) TFPOT $\underline{\mathrm{a}}$

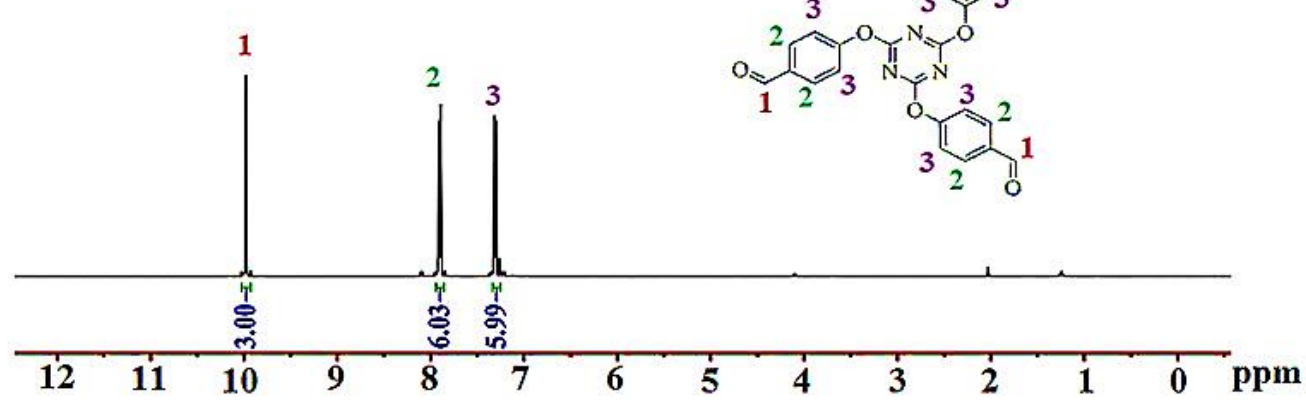

b.

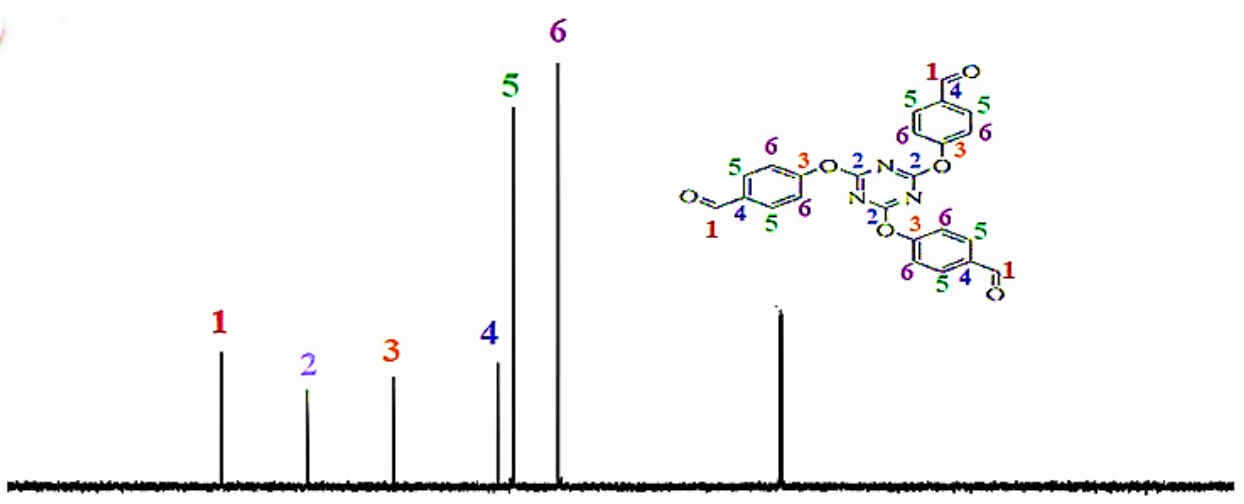

Figure S2: (a) ${ }^{1} \mathrm{H}$ NMR and (b) ${ }^{13} \mathrm{C}$ NMR spectra of synthesized TFPOT 
Table S1. Identification analysis to characterize the TFPOT structure.

\begin{tabular}{|c|c|c|c|c|}
\hline \multirow{11}{*}{ NMR } & \multirow{4}{*}{$\begin{array}{l}{ }^{1} \mathrm{H} \mathrm{NMR} \\
\left(\mathrm{CDCl}_{3}\right)\end{array}$} & Hydrogen type & $\sigma$ & $\begin{array}{c}\text { The number of } \\
\text { hydrogen }\end{array}$ \\
\hline & & $\mathrm{Ar}-\mathrm{H}$ & 7.26 & 6 \\
\hline & & $\mathrm{Ar}-\mathrm{H}$ & 7.86 & 6 \\
\hline & & $-\mathrm{CHO}$ & 9.95 & 3 \\
\hline & \multirow{7}{*}{$\begin{array}{c}{ }^{13} \mathrm{C} \mathrm{NMR} \\
\left(\mathrm{CDCl}_{3}\right)\end{array}$} & Carbon type & & $\sigma$ \\
\hline & & C6 & & 122 \\
\hline & & $\mathrm{C} 5$ & & 130.8 \\
\hline & & $\mathrm{C} 4$ & & 133.9 \\
\hline & & C3 & & 155.4 \\
\hline & & $\mathrm{C} 2$ & & 172.6 \\
\hline & & $\mathrm{C} 1$ & & 190.6 \\
\hline \multirow{3}{*}{ CHNS } & \multirow{3}{*}{$\begin{array}{l}\text { Anal. Calcd. for } \\
\mathrm{C}_{24} \mathrm{H}_{15} \mathrm{~N}_{3} \mathrm{O}_{6} \\
\text { Found }\end{array}$} & $\mathrm{C}(\%)$ & $\mathrm{N}(\%)$ & $\mathrm{H}(\%)$ \\
\hline & & 65.31 & 9.52 & 3.43 \\
\hline & & 65.04 & 3.75 & 9.44 \\
\hline
\end{tabular}

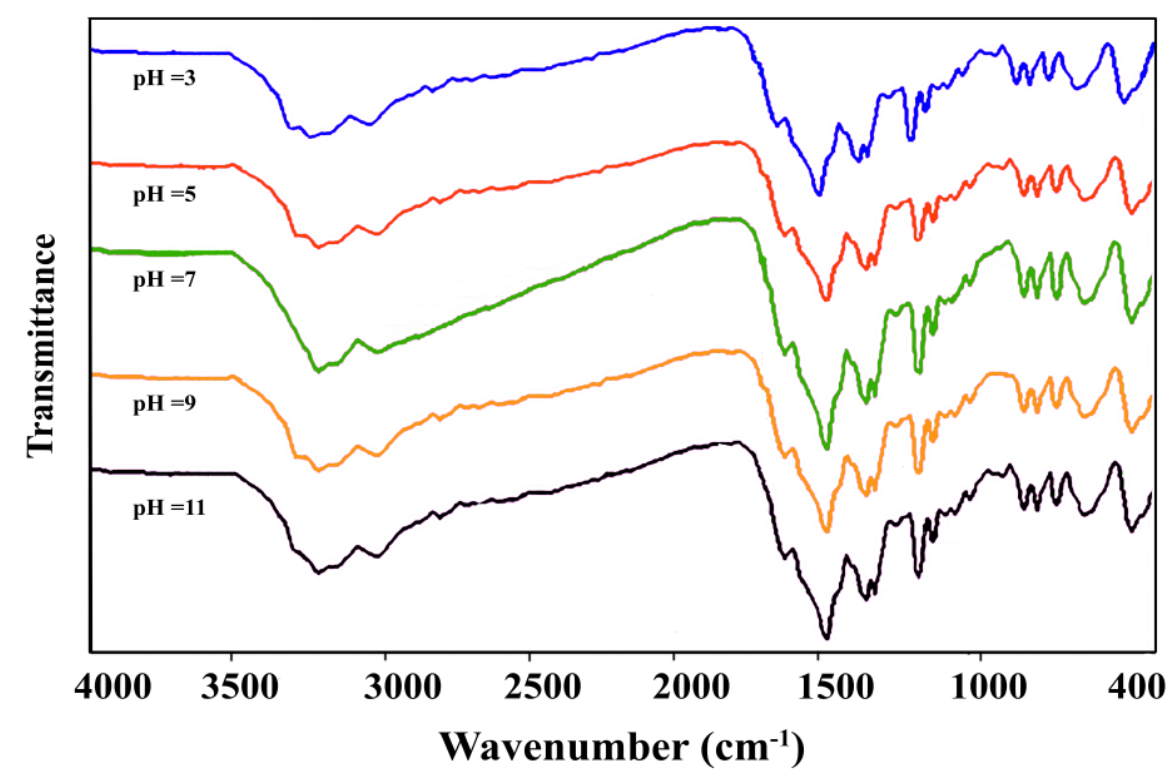

Figure S3. Evaluation of T-COF stability in various $\mathrm{pH}$ conditions 


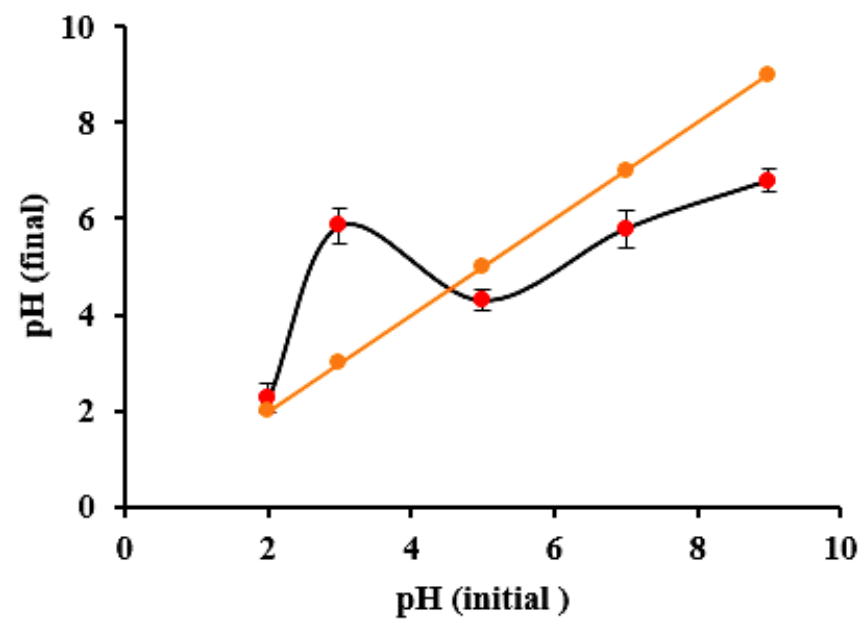

Figure S4: The variation in final $\mathrm{pH}$ as a function of initial $\mathrm{pH}$ onto the $\mathrm{T}-\mathrm{COF}$ suspension in water during the determination of the $\mathrm{pH}_{\mathrm{pzc}}$.

(a)

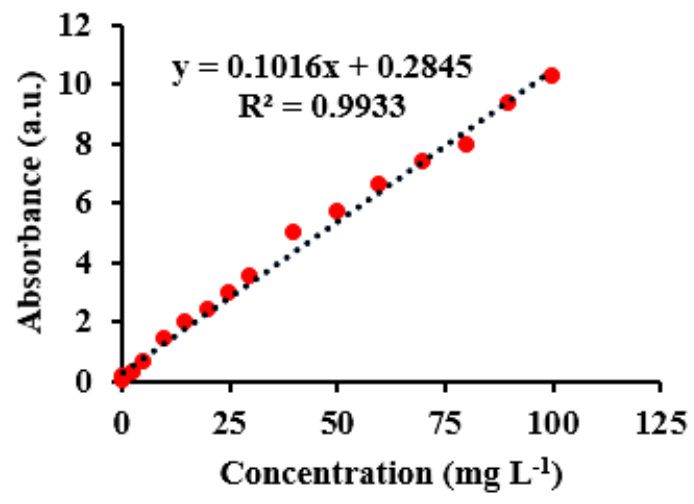

(c)

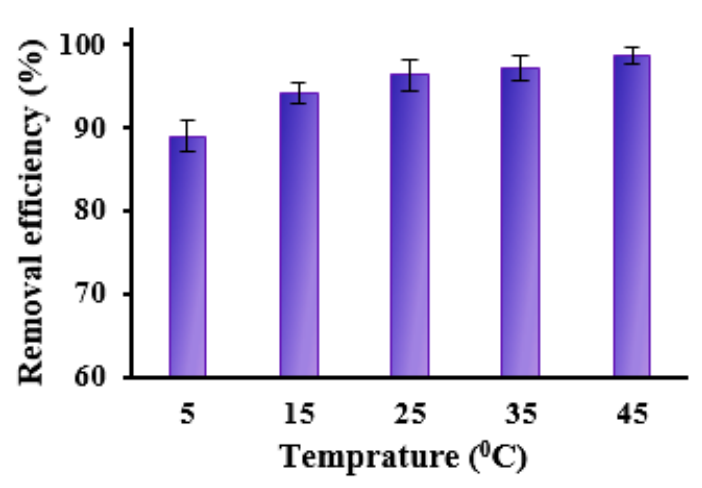

(b)

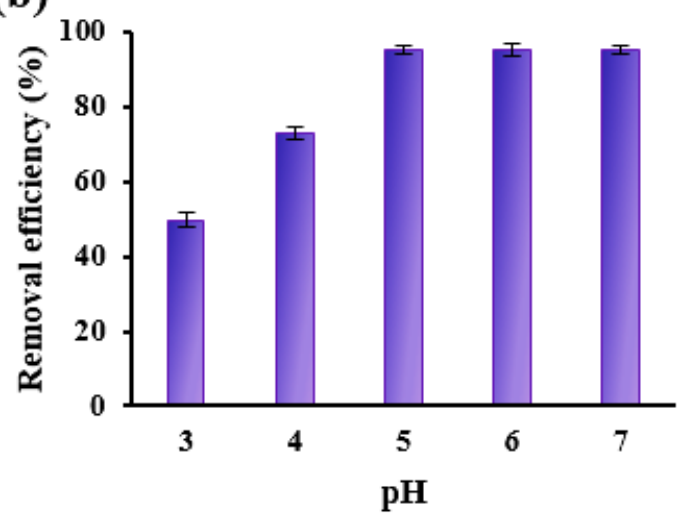

(d)

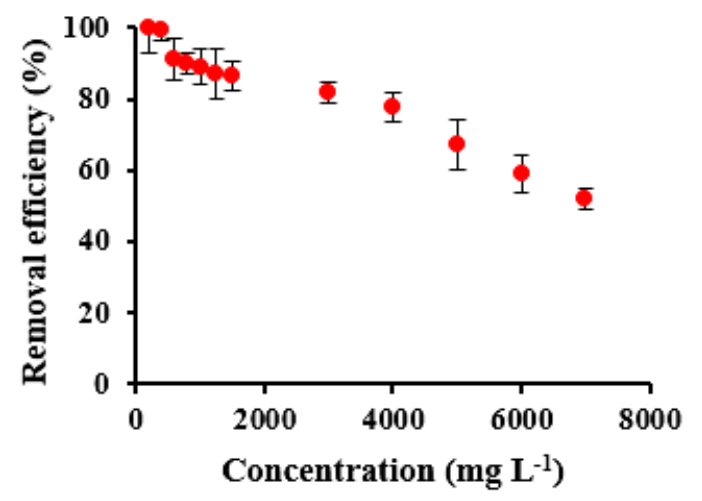


Figure S5. (a) Calibration curve for $\mathrm{Hg}^{2+}$-diphenylcarbazone complex, (b) Effect of $\mathrm{pH}$ on the elimination of $\mathrm{Hg}^{2+}$ ions by $\mathrm{T}-\mathrm{COF}$, (c) Effect of temperature on removal of $\mathrm{Hg}^{2+}$ ions using $\mathrm{T}$ $\mathrm{COF}$, (d) Effect of various $\mathrm{Hg}^{2+}$ concentration in removal efficiency (all of the data obtained from UV-vis spectrometer.

Table S2. Resulting Parameters from UV-vis Spectrometer for $\mathrm{Hg}^{2+}$ Adsorption on T-COF.

\begin{tabular}{|c|c|c|c|c|c|c|c|c|c|}
\hline \multirow{2}{*}{$\begin{array}{l}\text { isotherm } \\
\text { parameter }\end{array}$} & \multicolumn{3}{|c|}{ Tempkin } & \multicolumn{3}{|c|}{ Longmuir } & \multicolumn{3}{|c|}{ Freundlich } \\
\hline & $K_{T}$ & $b_{\mathrm{T}}$ & $R^{2}$ & $q_{\max }($ cal. $)$ & $b$ & $R^{2}$ & $K_{F}$ & $n$ & $R^{2}$ \\
\hline linear & 0.0292 & 5.900 & 0.977 & 2000 & 0.0027 & 0.998 & 48.07 & 2.889 & 0.956 \\
\hline nonlinear & 0.0292 & 5.900 & 0.977 & 2076 & 0.0025 & 0.996 & 94.27 & 2.645 & 0.929 \\
\hline
\end{tabular}
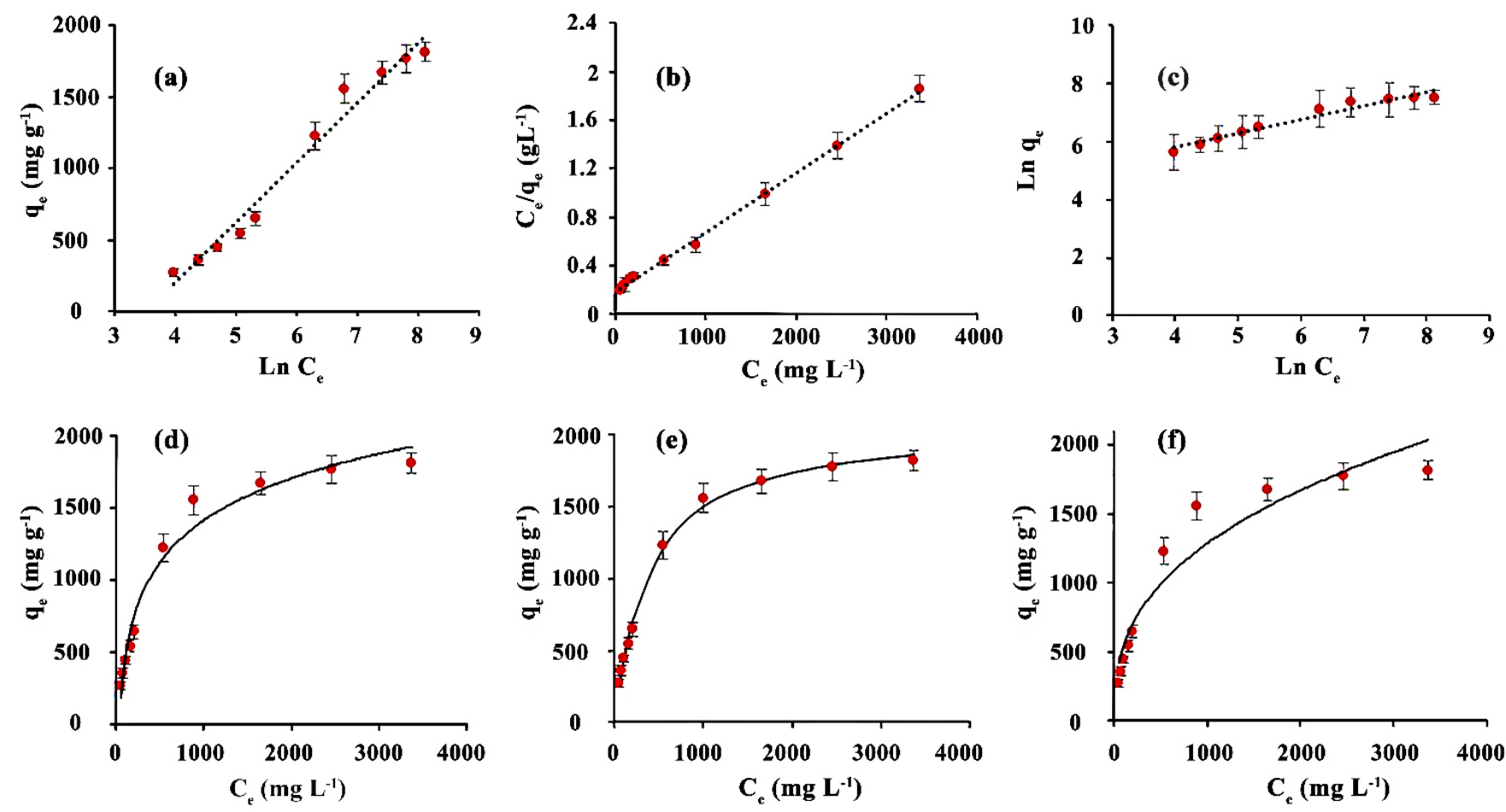

Figure S6. Linear and nonlinear forms of Tempkin (a, d), Langmuir (b, e), and Freundlich (c, d), for T-COF adsorption isotherms obtained from UV-vis spectrometer data. 
Table S3. Resulting Parameters from UV-vis Spectrometer for Kinetic $\mathrm{Hg}^{2+}$ Adsorption.

\begin{tabular}{|c|c|c|c|c|c|c|c|c|c|}
\hline \multirow{2}{*}{$\begin{array}{l}\text { model } \\
\text { parameter }\end{array}$} & \multicolumn{3}{|c|}{ pseudo-first-order } & \multicolumn{3}{|c|}{ pseudo-second-order } & \multicolumn{3}{|c|}{ intraparticle } \\
\hline & $q_{\mathrm{e}}($ cal. $)$ & $K_{1}$ & $R^{2}$ & $q_{\mathrm{e}}($ cal. $)$ & $K_{2}$ & $R^{2}$ & $K_{\text {id }}$ & $I$ & $R^{2}$ \\
\hline linear & 64.80 & 0.0076 & 0.870 & 243.9 & 0.0006 & 0.999 & 6.469 & 133.7 & 0.498 \\
\hline nonlinear & 231.8 & 0.1034 & 0.906 & 247.6 & 0.0006 & 0.882 & 15.68 & - & 0.428 \\
\hline
\end{tabular}
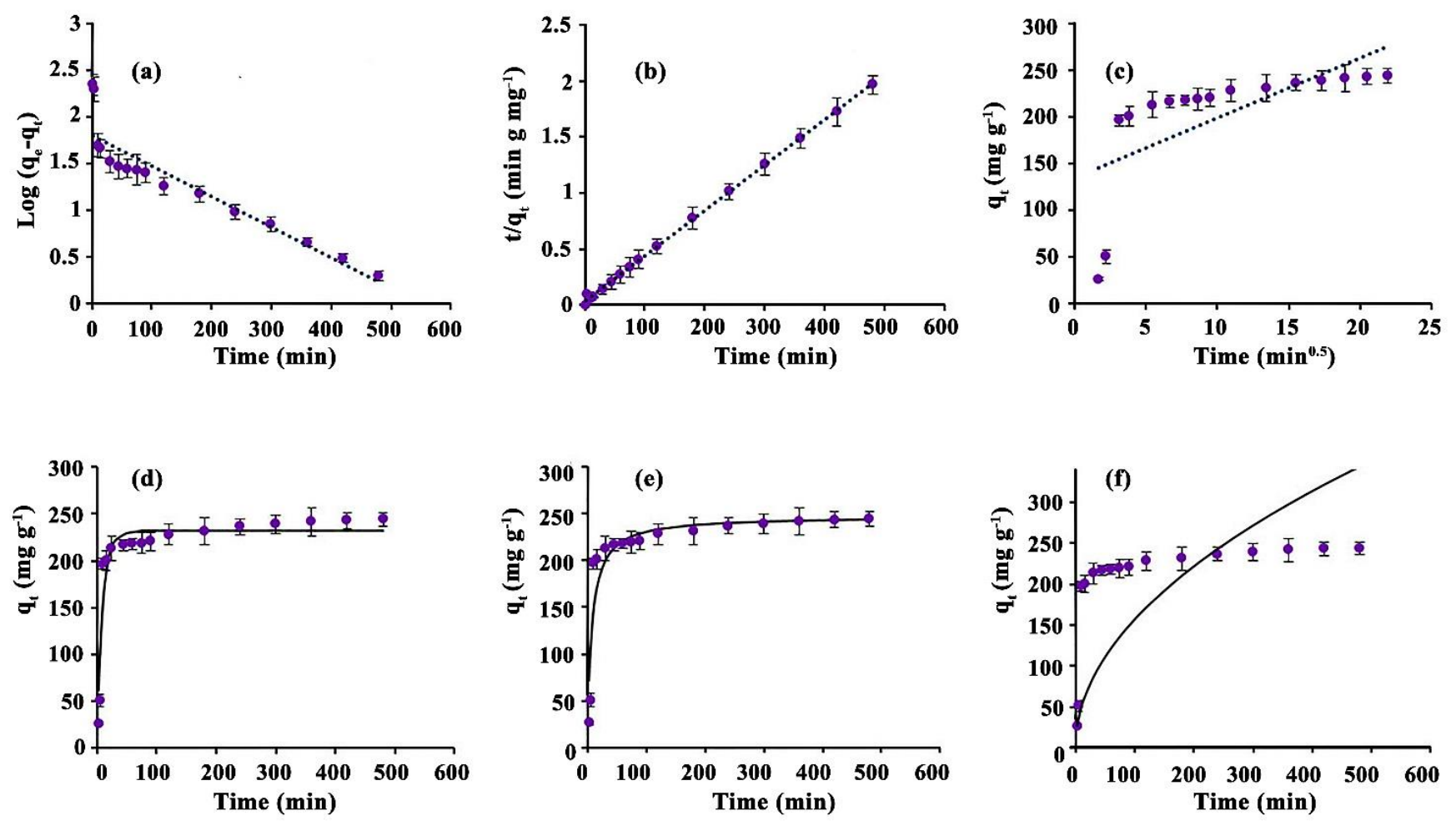

Figure S7. Linear and nonlinear forms of pseudo-first-order (a, d), pseudo-second-order (b, e), and Intraparticle (c, d), Kinetic models for $\mathrm{Hg}^{2+}$ adsorption on T-COF of obtained data from UVvis spectrometer. 